\title{
Ultrasound elastography is a reproducible and feasible tool for the evaluation of the patellar tendon in healthy subjects
}

\author{
F. PORTA, ${ }^{1}$ N. DAMJANOV,${ }^{2}$ F. GALLUCCIO,${ }^{1}$ A. IAGNOCCO ${ }^{3}$ and \\ M. MATUCCI-CERINIC ${ }^{1}$ \\ ${ }^{1}$ Division of Rheumatology AOUC, Department of Sperimental and Clinical Medicine, University of Florence, Florence, Italy, \\ ${ }^{2}$ Institute of Rheumatology, University of Belgrade School of Medicine, Belgrade, Serbia, and ${ }^{3}$ Dipartimento Medicina Interna \\ e Specialità Mediche: Reumatologia, Sapienza Università di Roma, Rome, Italy
}

\begin{abstract}
Objective: To evaluate the feasibility and reproducibility of ultrasound elastography (UE) in the assessment of healthy patellar tendon and to describe its UE pattern.

Methods: Twenty-two patellar tendons of 11 out of 16 healthy subjects who met the inclusion criteria were evaluated three times by ultrasound (US) and UE at their proximal, middle and distal portions, by two separate sonographers with different experiences in UE.

Results: In all tendon portions the color map analysis showed a predominance of green (highly elastic), with good values of intra-observer (Operator $1: P$-values $=0.790,0.864,0.865$; Operator $2: P=0.642,0.882,0.613$ for proximal, middle and distal portions, respectively $)$ and inter-observer $(P=0.657)$ agreement. For both operators the intra-observer analysis of the elasticity ratio (ER) between the tendon and the subcutis showed high agreement values $(P<0.001$ for both operators). The inter-observer analysis showed also high agreement values ( $P<0.001$ at proximal, $P=0.001$ at middle, $P=0.005$ at distal portions). The overall analysis of the ER of the tendon portions showed values of (mean $\pm \mathrm{SD}$ ): $1.47 \pm 0.64,4.38 \pm 1.36,3.32 \pm 1.20$ for proximal, middle and distal portions, respectively. The mean time to perform the UE evaluation for the inexperienced operator was $5 \mathrm{~min}$ at the beginning of the study but decreased to $2 \mathrm{~min}$ after a few examinations were done. The mean time for the expert was 2 min for the entire study.

Conclusions: UE is a feasible and reproducible tool for the evaluation of the healthy patellar tendon and further data are needed to define its role in the assessment of tendon pathology.
\end{abstract}

Key words: feasibility, healthy subjects, patellar tendon, reproducibility, ultrasound elastography.

\section{INTRODUCTION}

Ultrasound (US) has become the reference tool for a rapid and reliable musculoskeletal evaluation. In fact, its low cost and easy access as well as noninvasiveness and dynamic examination, have made US a friendly

Correspondence: Francesco Porta, MD, Division of Rheumatology AOUC, Department of Biomedicine, Villa Monna Tessa, viale Pieraccini 18, 50139 Firenze, Italy.

Email: dr.fporta@gmail.com and useful technique for diagnosis and therapeutic follow-up. ${ }^{1,2}$ In rheumatology, US allows the evaluation of tendons with high frequency transducers to identify fine structural alterations. ${ }^{3}$ However, still today the recognition of a pathologic echo-texture in respect to the healthy surrounding tissue remains sometimes a challenge. ${ }^{3}$ The most widely used technique for the study of tendons is B-mode US, which provides morphological data only, ${ }^{4,5}$ while no information is given about biomechanical tissue properties. However, the evaluation of biomechanical characteristics may give more details 
on the functionality and status of tendons due to inflammation or degenerative conditions.

Ultrasound elastography (UE) is a new dynamic technique for the assessment of the biomechanical properties of soft tissues. It estimates the stiffness by measuring the entity of tissue strain after application of an external compression. ${ }^{6}$ UE is performed by applying local repetitive compressions at the area of interest by using the US probe, which induces tissue deformations. The most commonly used UE technique is based on the execution of manual freehand movements, which produces a color scale map that is superimposed on the B-mode image. The different colors express different elasticity degrees, usually varying from red (highly elastic tissue) to blue (barely elastic tissue) while the other colors represent intermediate degrees of stiffness. ${ }^{7}$

UE has already been employed for the evaluation of Achille's tendon and common extensor tendon. In almost all papers the healthy tendons appeared very hard while in cases of pathological alterations a softening was detected. ${ }^{8-12} \mathrm{~A}$ common feature of these works is that they evaluate a tendon connecting a muscle to a bone, while no data are present in the literature about the appearance of ligaments and tendons connecting two bones (as the patellar tendon).

The aim of this study was to evaluate the feasibility and reproducibility of UE in the assessment of healthy patellar tendon, by measuring the inter- and intraobserver variations and to describe the UE pattern of the healthy patellar tendon.

\section{METHODS}

A total of 16 healthy subjects were studied; exclusion criteria were previous trauma, psoriasis, dismetabolism, agonistic sport activities, connective tissue diseases and a body mass index (BMI) higher than 25 . The presence of patellar tendon alterations on B-mode scan represented an exclusion criterion for the subsequent UE evaluation.

Ethics committee approval was obtained from our institution and all the healthy volunteers signed informed consent forms.

\section{US and UE evaluation}

Sonographic evaluations were carried out using an ESAOTE MyLab70 Gold XV with a 6-18 MHz linear probe (with fixed setting at $15 \mathrm{MHz}$ ) and by applying the elastographic module (UE).

All subjects underwent transverse and longitudinal US scan of both patellar tendons with the knee flexed at $30^{\circ}$. At all sites the tendon echo-texture and thickness (measured both at the distal and proximal entheseal levels and at the middle part of the tendon), the presence of enthesophytes, erosion and bursal abnormalities were evaluated.

UE evaluation of the patellar tendons was carried out by two operators (FP, ND) with at least 8 years of experience in musculoskeletal US, and was performed at the level of the proximal (patellar enthesis), middle and distal portion (tibial enthesis) only in those subjects who did not present any abnormalities on the B-mode assessment.

To obtain UE images, repetitive free-hand movements of compression and decompression of the probe over the area of interest were carried out (amplitude approximately less than $2 \mathrm{~mm}$; frequency of 1-2 movements per second). During the UE evaluation, the reference B-mode image was maintained. A fixed depth of $3 \mathrm{~cm}$ was set for the B-mode evaluation, while a fixed depth of $1.5 \mathrm{~cm}$ with a span covering the whole B-mode area was set for UE assessment. The UE images were characterized by a color translucent map superimposed on the B-mode images, in which each color indicated the relative elasticity of the tendon compared to the mean elasticity of the whole examined area (in our machine, from red $=$ barely elastic structure, to violet $=$ intermediate elastic tissue, to green $=$ highly elastic structure).

The elasticity ratio (ER), which is the ratio between the elasticity of two regions of interest (ROI) has been calculated between the subcutaneous tissue and the tendon (Fig. 1a-c).

Every tendon portion (proximal, middle and distal) was evaluated by two separate and blinded operators, expert in musculoskeletal US but with different levels of experience in UE (operator $1=\mathrm{FP}, 1$ month training with at least 50 evaluations; operator $2=\mathrm{ND}, 1$ day training with five evaluation) to calculate the interobserver agreement. Each measurement was repeated three times to calculate intra-observer agreement.

The parameters that have been considered for the US assessment were the tendon thickness and shape, the echo-texture and the presence of tears.

The parameters considered for the UE were the predominant color and the elasticity ratio compared to the subcutaneous tissue.

\section{Statistical analysis}

The evaluation of intra-observer and inter-observer agreement, expressed as reliability, correlation coefficient and confidence range, have been done with Friedman and Wilcoxon tests, respectively. The values of 


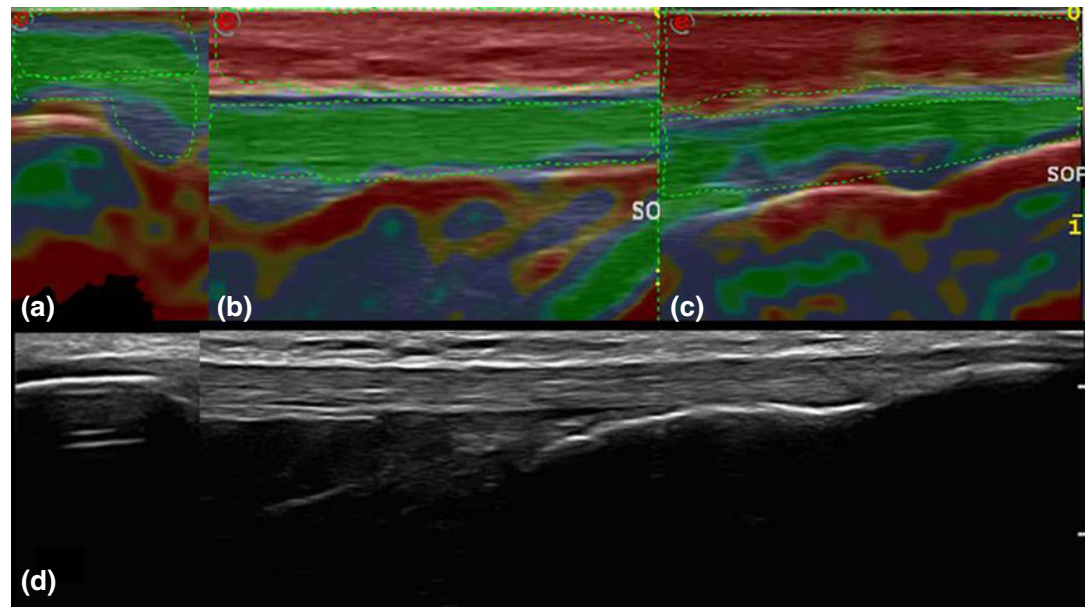

Figure 1 Ultrasound elastography (UE) colorimetric analysis of the proximal (a), middle (b) and distal (c) portions of the patellar tendon and panoramic B-mode view of the patellar tendon $(\mathrm{d})$. In $\mathrm{a}, \mathrm{b}$ and $\mathrm{c}$ the dotted lines indicate the ROIs to calculate the elasticity ratio (ER). elasticity have been expressed as mean values, standard deviation and range.

\section{RESULTS}

Out of 16 subjects examined by US, five were excluded due to the presence of patellar tendon alterations on B-mode scan (two subjects with a small hypoechoic area due to partial lesion, one with monolateral proximal entesopathy, one with small proximal entesophytes and one with small bilateral distal bursitis), while 11 subjects (seven female, four male; mean age 24, age range 21-34 years underwent UE bilateral examination of the patellar tendons.

A total of 22 tendons were evaluated with UE by two operators in the proximal, middle and distal portions of the patellar tendon, repeating each evaluation in three different times (total of 198 examinations per each operator).

In the proximal portion, the patellar enthesis, the subcutis and the superficial half of the tendon were green while the deep layer was violet and the underlying bone cortex was red (Fig 1a). In the middle portion the subcutis was red, the tendon uniformly green and the superficial part of the Hoffa fat pad red (Fig. 1b). In the distal part, in the tibial enthesis, the subcutis was red, the tendon was mostly green and the bone cortex red (Fig. 1c).

In each tendon portion the analysis of the color map showed a predominance of green (Fig. 1), with high values of intra-observer (operator 1: $P=0.790,0.864$, 0.865 ; operator 2: $P=0.642,0.882,0.613$ for proximal, middle and distal portions, respectively) and interobserver $(P=0.657)$ agreement.
For both operators the intra-observer analysis of the ER between the subcutis and the tendons showed high agreement values in the three tendon portions $(P<0.001$ for each operator in each tendon area $)$ (Table 1).

The inter-observer analysis of the ER in the three tendon portions showed high agreement values $(P<0.001$ for proximal, $P=0.001$ for middle, $P=0.005$ for distal) (Table 2).

The overall analysis of the ER of the three tendon portions showed values of (mean \pm SD) $1.47 \pm 0.64$ for the proximal area, $4.38 \pm 1.36$ for middle and $3.32 \pm 1.20$ for the distal area.

The mean time needed to perform a complete UE examination of a patellar tendon was $5 \mathrm{~min}$ at the beginning for the inexperienced operator, then it decreased to less than 2 min after a training of 10 tendon evaluations. The mean time for the more expert operator was less than $2 \mathrm{~min}$ for the entire study.

\section{DISCUSSION}

The present study is the first attempt to define the reproducibility and feasibility of UE in the evaluation of the healthy patellar tendon. Currently, US is widely used for tendon evaluation, being easily accessible, safe, inexpensive, reproducible and accurate. ${ }^{1}$ Despite this, some tendon abnormalities may be difficult to detect by B-mode US evaluation. Moreover, US does not provide information about the mechanical properties of the tendons. For this reason, new imaging techniques which are able to assess tendon structure as well as its mechanical properties, are an important step forward in the diagnostic imaging of these structures. Our data show a 
Table 1 Intra-observer analyses

\begin{tabular}{|c|c|c|c|c|c|c|c|}
\hline & $\begin{array}{c}\text { Minimum } \\
\text { ER }\end{array}$ & $\begin{array}{l}\text { Maximum } \\
\text { ER }\end{array}$ & Mean \pm SD & Reliability & ICC & $\begin{array}{c}\text { Confidence } \\
\text { range }\end{array}$ & $P$-value \\
\hline \multicolumn{8}{|l|}{ Operator 1} \\
\hline \multirow[t]{3}{*}{ Proximal } & 0.60 & 3.26 & $1.31 \pm 0.64$ & 0.919 & 0.790 & $0.627-0.898$ & $<0.001$ \\
\hline & 0.62 & 2.39 & $1.27 \pm 0.49$ & & & & \\
\hline & 0.53 & 2.19 & $1.22 \pm 0.40$ & & & & \\
\hline \multirow[t]{3}{*}{ Middle } & 2.17 & 6.96 & $4.59 \pm 1.49$ & 0.850 & 0.654 & $0.426-0.826$ & $<0.001$ \\
\hline & 1.85 & 7.15 & $4.73 \pm 1.46$ & & & & \\
\hline & 2.49 & 7.03 & $5.08 \pm 1.31$ & & & & \\
\hline \multirow[t]{3}{*}{ Distal } & 2.01 & 5.97 & $3.49 \pm 1.12$ & 0.878 & 0.705 & $0.502-0.852$ & $<0.001$ \\
\hline & 1.52 & 6.07 & $3.70 \pm 1.17$ & & & & \\
\hline & 1.34 & 6.32 & $3.84 \pm 1.35$ & & & & \\
\hline \multicolumn{8}{|l|}{ Operator 2} \\
\hline \multirow[t]{3}{*}{ Proximal } & 0.59 & 3.80 & $1.66 \pm 0.82$ & 0.899 & 0.749 & $0.564-0.876$ & $<0.001$ \\
\hline & 0.51 & 3.45 & $1.65 \pm 0.85$ & & & & \\
\hline & 0.74 & 3.21 & $1.67 \pm 0.70$ & & & & \\
\hline \multirow[t]{3}{*}{ Middle } & 1.49 & 6.00 & $3.86 \pm 1.36$ & 0.895 & 0.740 & $0.546-0.874$ & $<0.001$ \\
\hline & 1.17 & 6.72 & $4.05 \pm 1.54$ & & & & \\
\hline & 1.22 & 6.41 & $3.97 \pm 1.65$ & & & & \\
\hline \multirow[t]{3}{*}{ Distal } & 0.81 & 5.25 & $2.85 \pm 1.29$ & 0.857 & 0.666 & $0.442-0.833$ & $<0.001$ \\
\hline & 1.31 & 5.47 & $2.94 \pm 1.33$ & & & & \\
\hline & 0.75 & 6.57 & $3.17 \pm 1.51$ & & & & \\
\hline
\end{tabular}

ER, elasticity ratio; ICC, intraclass coefficient.

Table 2 Inter-observer reliability

\begin{tabular}{|c|c|c|c|}
\hline & Proximal & Middle & Distal \\
\hline \multicolumn{4}{|l|}{ Operator 1} \\
\hline Minimum ER & 0.73 & 2.33 & 1.62 \\
\hline Maximum ER & 2.61 & 6.81 & 5.84 \\
\hline Mean ER \pm SD & $1.27 \pm 0.48$ & $4.80 \pm 1.24$ & $3.68 \pm 1.09$ \\
\hline \multicolumn{4}{|l|}{ Operator 2} \\
\hline Minimum ER & 0.69 & 1.32 & 0.96 \\
\hline Maximum ER & 3.16 & 5.70 & 5.22 \\
\hline Mean ER \pm SD & $1.66 \pm 0.72$ & $3.96 \pm 1.37$ & $2.97 \pm 1.22$ \\
\hline \multicolumn{4}{|l|}{ Inter-observer } \\
\hline Reliability & 0.783 & 0.748 & 0.690 \\
\hline ICC & 0.644 & 0.597 & 0.527 \\
\hline $\begin{array}{l}\text { Confidence } \\
\text { range }\end{array}$ & $0.314-0.835$ & $0.244-0.811$ & $0.145-0.772$ \\
\hline$P$-value & $<0.001$ & 0.001 & 0.005 \\
\hline
\end{tabular}

ER, elasticity ratio; ICC, intraclass coefficient.

good inter-observer and intra-observer agreement for UE evaluation of the healthy patellar tendon. The agreement between the experienced and inexperienced UE operators suggests that the technique is easy to perform and quick to learn.

The time needed to carry out a complete UE examination of a patellar tendon was approximately $5 \mathrm{~min}$ at the beginning of the study for the inexperienced operator, but it has lowered to $2 \mathrm{~min}$ after the first five examinations, thus confirming the feasibility of the technique.

Our data show a characteristic UE pattern for the three tendon portions; in each tendon portion the predominant color was green, which corresponds to highly elastic structure. This result is apparently in contrast with the data obtained in the previous studies, where the healthy tendon structure appeared very stiff, while a softening was detected in cases of pathological alterations. $^{8-12}$ This discrepancy can be explained considering that we evaluated the patellar tendon, which connects two hard and fix structures (patellar bone and tibial tuberosity), while in the other papers, the authors evaluated tendons attached to a hard and fixed structure on one side (bone) and to a soft and compliant structure (muscle) on the other. Considering that elasticity is defined as the capacity of a structure to resist a deformation by an external force and to get back to the initial shape once the force is ended, the damper power of the muscle can be responsible for the loss of elasticity (i.e. the capacity to get back to the initial shape) of the whole system, explaining why tendons connecting a muscle to a bone results in inelasticity, while a tendon connecting two bones appears highly elastic. 
These preliminary data obtained in young healthy subjects seem to be encouraging about the possibility to define a "normal tendon UE pattern" but they have been found in a small number of subjects and need to be confirmed on a wider population, including older subjects, in order to define the influence of the age on the tissue echotexture.

In the future, together with conventional US evaluation, UE of tendons could be applied to detect many conditions such as enthesopathy, tendinopathy, small lesions, overuse alterations and inflammation as well as modification of elastic properties of tendons not associated with changes in echotexture.

In this work, a numeric value was given to describe the elasticity of the tendon structure in comparison to the subcutis. This permits a quantitative evaluation of the tendon elastic properties in comparison to the colorimetric qualitative evaluation, thus allowing us to define numeric ranges of normality.

In conclusion, UE of the patellar tendon is a feasible and reproducible tool in the assessment of the healthy patellar tendon. Further data are needed to define its role in pathologic modifications of tendons.

\section{DISCLOSURE STATEMENT}

For all the authors no additional funding was received and there are no conflicts of interest, both personal and/or institutional, that are relevant to the work conducted or reported in this manuscript.

\section{REFERENCES}

1 Wakefield RJ, Gibbon WW, Emery P (1999) The current status of ultrasonography in rheumatology. Rheumatology (Oxford) 38 (3), 195-8.
2 Kane D, Balint PV, Sturrock R, Grassi W (2004) Musculoskeletal ultrasound-a state of the art review in rheumatology. Part 1: current controversies and issues in the development of musculoskeletal ultrasound in rheumatology. Rheumatology (Oxford) 43, 823-8.

3 Martinoli C, Bianchi S, Dahmane M et al. (2002) Ultrasound of tendons and nerves. Eur Radiol 12 (1), 44-55.

4 Fornage BD, Rifkin MD (1988) Ultrasound examination of tendons. Radiol Clin North Am 26 (1), 87-107.

5 Robinson P (2009) Sonography of common tendon injuries. AJR Am J Roentgenol 193, 607-18.

6 Ophir J, Alam SK, Garra B et al. (1999) Elastography: ultrasonic estimation and imaging of the elastic properties of tissues. Proc Inst Mech Eng H 213, 203-33.

7 Iagnocco A, Kaloudi O, Perella C et al. (2010) Ultrasound elastography assessment of skin involvement in systemic sclerosis: lights and shadows. J Rheumatol 37, 1688-91.

8 Tan S, Kudaş S, Özcan AS et al. (2012) Real-time sonoelastography of the Achilles tendon: pattern description in healthy subjects and patients with surgically repaired complete ruptures. Skeletal Radiol 41, 1067-72.

9 De Zordo T, Chhem R, Smekal V et al. (2010) Real-time sonoelastography: findings in patients with symptomatic achilles tendons and comparison to healthy volunteers. Ultraschall Med 31, 394-400.

10 Drakonaki EE, Allen GM, Wilson DJ (2009) Real-time ultrasound elastography of the normal Achilles tendon: reproducibility and pattern description. Clin Radiol 64, 1196-202.

11 De Zordo T, Fink C, Feuchtner GM, Smekal V, Reindl M, Klauser AS (2009) Real-time sonoelastography findings in healthy Achilles tendons. AJR Am J Roentgenol 193, W134-8.

12 De Zordo T, Lill SR, Fink C et al. (2009) Real-time sonoelastography of lateral epicondylitis: comparison of findings between patients and healthy volunteers. AJR Am J Roentgenol 193 (1), 180-5. 